\title{
Mediating Everyday Data Encounters with Mundane Video Vignettes
}

\author{
Yu Zhang \\ STUDIO Ü, Eindhoven, the Netherlands \\ yuzhang.nl@gmail.com
}

\begin{abstract}
Data in everyday life is represented in ways that are supposed to connect with our needs, tasks and situations, to inform and facilitate effectiveness and functionalism. And still, all we see are numbers, charts or graphics. We engage in sense-making all day through analytical interpretation, which is often not intuitive and causes friction whenever we encounter it in an everyday context. In our work, we aim for a different experience in everyday data-sensemaking that translates sensor data, time and data from external sources into lively, yet mundane video vignettes. The aim is to connect the experience to the phenomenon behind data, to deny data the stage and spotlight in our Everyday. This case study shows the design and implementation of a novel, interactive video installation that projects mundane data into an Everyday context, giving the data a new form and meaning. We work with video vignettes that are designed as short, neutral video fragments and show a person in different mundane situations, acting alone or interacting with props. The design process went through three iterations before the installation was exhibited as part of a group exhibition. We reflect on the most important design decisions, turning points in the process and how design and implementation are intertwined throughout the installation preparation. We conclude with a short overview of the contribution and an outlook to future work.
\end{abstract}

\section{CCS CONCEPTS}

- Human-centered computing $\rightarrow$ Visualization; Empirical studies in visualization; $\bullet$ Applied computing $\rightarrow$ Arts and humanities; Media arts.

\section{KEYWORDS}

Data representation, data scenarios, everyday data, video vignette, personal narrative, video installation, interaction design, media arts

\section{ACM Reference Format:}

Yu Zhang and Mathias Funk. 2021. Mediating Everyday Data Encounters with Mundane Video Vignettes. In CHI Conference on Human Factors in Computing Systems Extended Abstracts (CHI '21 Extended Abstracts), May 08-13, 2021, Yokohama, Japan. ACM, New York, NY, USA, 9 pages. https: //doi.org/10.1145/3411763.3443433

Permission to make digital or hard copies of part or all of this work for personal or classroom use is granted without fee provided that copies are not made or distributed for profit or commercial advantage and that copies bear this notice and the full citation on the first page. Copyrights for third-party components of this work must be honored. For all other uses, contact the owner/author(s).

CHI '21 Extended Abstracts, May 08-13, 2021, Yokohama, Japan

(C) 2021 Copyright held by the owner/author(s)

ACM ISBN 978-1-4503-8095-9/21/05.

https://doi.org/10.1145/3411763.3443433

\author{
Mathias Funk \\ Eindhoven University of Technology, Eindhoven, the \\ Netherlands \\ m.funk@tue.nl
}

\section{INTRODUCTION}

Look around you, spot the sensors, screens and data in your direct proximity [27]. Every time you let your eyes wander, digits and graphs jump at you and update your perception of temperature, time and tasks with accurate data. Beyond your personal space, many applications are designed for representing everyday data [26]. They are designed in ways that can assist users to approach, consume and interpret data as absolute, precise and accurate information [7]. They provide the information that tries to connect with needs, tasks and situations, to achieve the aim of amplifying cognition and functionalism. Multiple applications or devices are gathering data from daily life all the time and visualize it in various ways for users. From web-based applications on everyday movement [13, 38] or energy consumption [23, 39] and air quality [28] in a home, to wearable sensors for detecting health information $[33,43]$, or security-focused smart home system [10, 35] that can offer more safety, ultimately, we expect everyday data [32,37] to be in these formats, details, forms of provision. The machines' accuracy and pace determine our expectations and perception of lived reality: users keep receiving and checking such non-stop information and do not even realize if it is uncertain $[5,17]$ or redundant. In contrast, most people who experience a weekend or holiday off this wiry race, report first a feeling of being disconnected, then a pleasant calm, a sense of timelessness. We can find examples in calm or slow interaction design $[18,19,30]$ that point in a similar direction, emphasizing reflection [46] or idleness [31]. There are some examples of experimental design that have explored the use of shape-changing interfaces for visualizing daily data. Those designs support an abstract representation of personal data in everyday life $[20,22]$ and they claim that data physicalizations can provide a seamless way for users to understand data [42, 44]. Everyday data in those works has often been represented as more abstract dynamics of physical artifacts and still needs human interpretation to make sense of. The question remains: will switching to a different output modality, raising the abstraction level, or adding metaphorical packaging substantially improve living with data? We think not. Studies have shown that fatigue and disinterest-or anxiety [36]-are plausible negative reactions to ubiquitous data and information displays [38]-and a lack of contextualization (e.g., in relation to big data [1]) and meaning seem to be factors in discontinued use. There is a notable gap in research on everyday data, ubiquitous representation and appropriate (interaction) design.

In this work, we explore a different take on peripheral data representations: we move away from accuracy and precise numbers and use this opening for contextualization that is based on personal narratives to achieve more intuitive interpretation of experiences in data visualization [16, 24]. "The 'homo narrans' theory assumes 


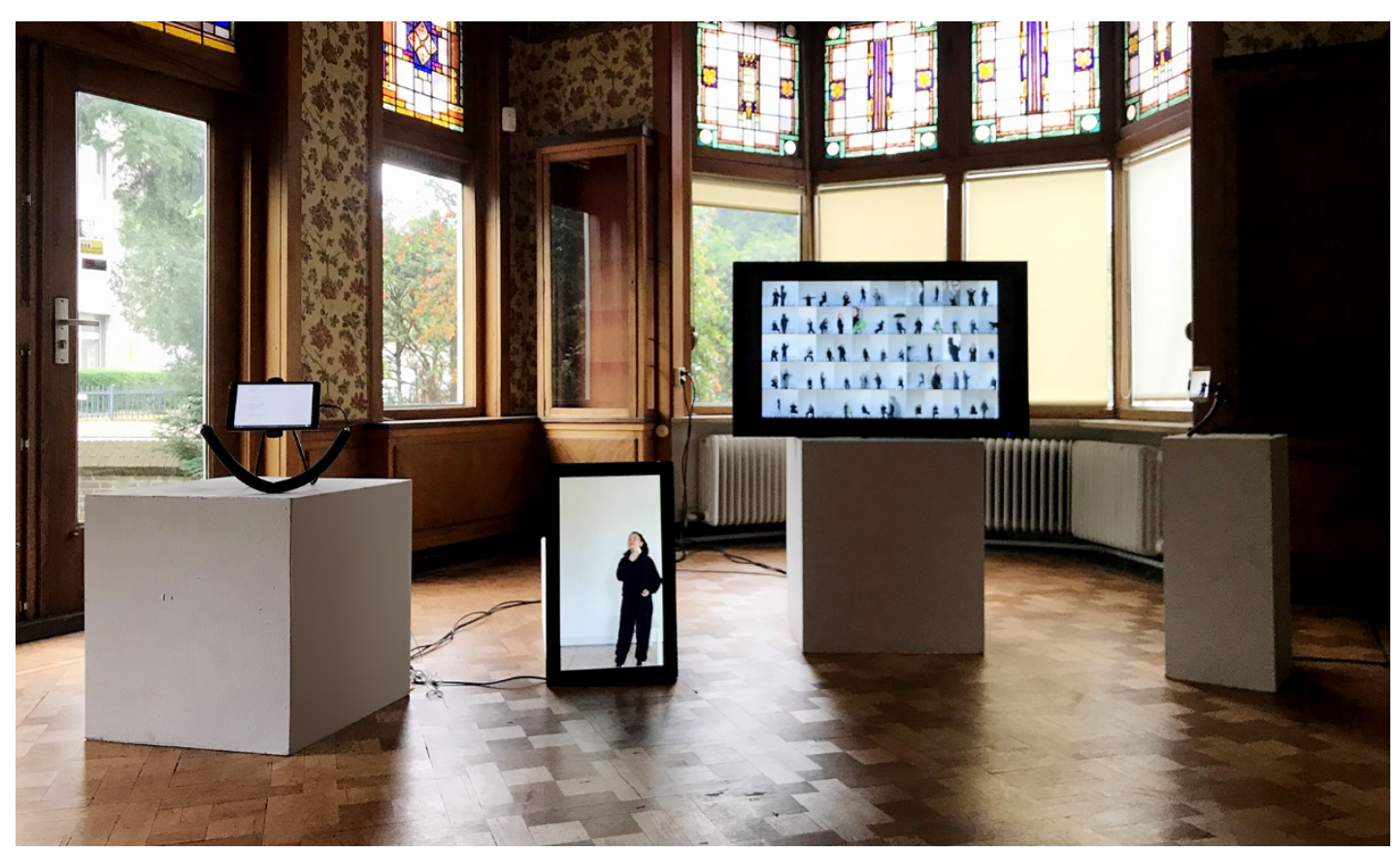

Figure 1: Exhibition view from LOST IN ILLUSION at Albert van Abbehuis, Eindhoven, the Netherlands. From left to right, internal data display (iPad), large video vignette (portrait standing display), vignette queue (large landscape display), and small video vignette (iPod touch, same as the large one). Sensors are not shown in this view.

that personal narratives can be an elementary human need and the most common form of discourse in everyday life." [8, 25]. From a cognitive perspective, the way of representing daily data is not only to associate with informing us about our everyday environment [40], but to integrate with relevant narratives as well $[8,29]$. We designed an interactive video installation (Figure 1) that has the explicit goal of challenging preconception of everyday data and its current representation methods in design. The resulting installation offers an emotional experience [45] through representing data or reviewing "memories through storytelling the daily data" [41]. For the users (named as "the visitors" in our case), the process of perceiving information is designed as a series of video vignettes, essentially glimpses of personal narratives, all with the objective to let them experience narrative empathy rather than leaving them with the task of analyzing, interpreting and internalizing abstract data. The point of the visitors' experience is to connect intuitively to what the data are about, not the data itself. The personal narrative that is visualized through the video vignette approximates plausibly how the visitors will react to or will be affected by the measured real-life phenomenon.

\section{DESIGN}

Our design process started with the given intent-designing a new, more intuitive, and more relatable experience that represents everyday data: as an interactive, multi-faceted video installation. In this section, we describe the design process that evolved to more accurate framings of data in the installation and also the additional intermediate concepts of data scenarios, personal narratives, video vignettes, as well as the transformations between them. Our design process consisted of three iterations and hinged on the general process of translating data into video vignettes, refining different parts of this translation in every iteration: (1) from data to desired interaction and experience (high-level and end-to-end translation), (2) from data to data scenarios to personal narratives (conceptual development of installation modules), (3) refining raw data and scenario processing (detailed design of installation modules). In the following, we will describe how we moved from the original design intent (iteration 1) to the more elaborate high-level design (iteration 2 ) to the final installation (iteration 3 and implementation). The design process is shown in Figure 2 (vertical axis) as a process of concept expansion and development.

\subsection{Iteration 1: from everyday data to desired experience}

Based on our original intent, the first iteration (see Figure 2) introduced personal narratives to achieve a desired experience that could let visitors of the installation empathize with the real-life phenomenon behind the data. Personal narratives helped bridge the gap from data to the desired experience.

2.1.1 From everyday data to personal narratives. Both everyday data and personal narratives link to real-life phenomena. For example, given a warm room in the summer, measuring the temperature data of "32 degrees Celsius" corresponds to a personal narrative of feeling warm, dressing light or drinking a cold beverage. In our case study, we selected six types of data - temperature, humidity, time, sound, proximity, and human presence from our everyday life environment - that included both environmental conditions 


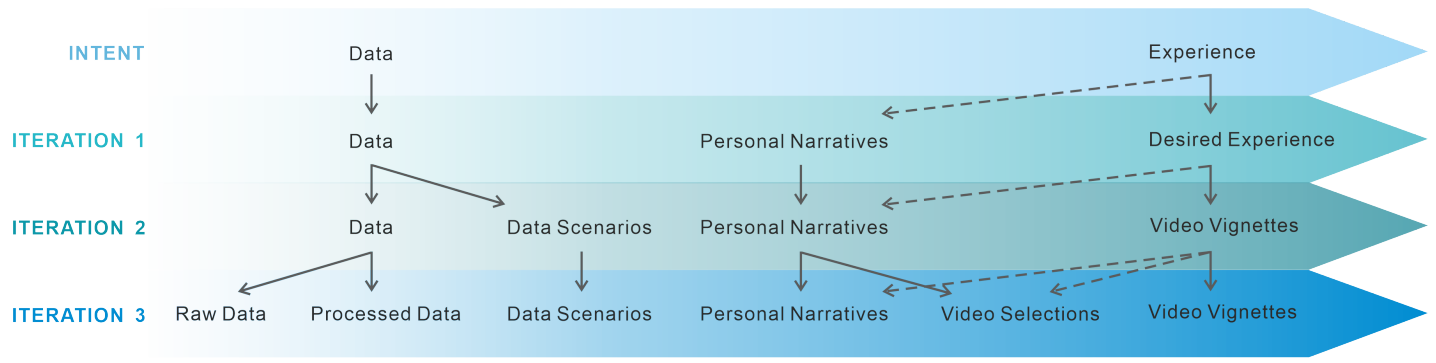

Figure 2: Overview of design process in this case study: initial intent and three iterations (top to bottom), data processing flow (left to right) and the different concepts we introduced. Edges show concept expansion and development (solid), and experienced relations (dashed).

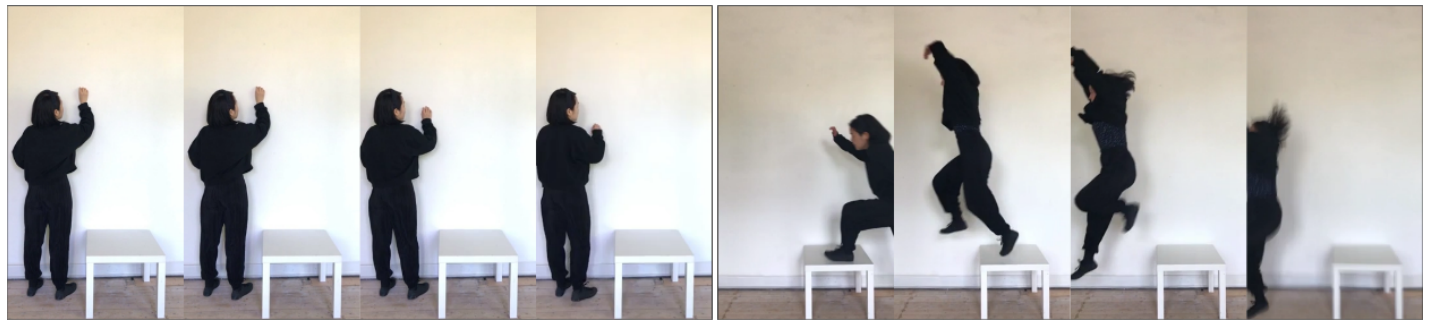

Figure 3: Movements and gestures from two video examples that designed for "non-active" data scenarios (first four images from left to right) and "active" data scenarios (second four images from left to right).

and human impacts. Apart from the situational relatedness, both everyday data and personal narratives share predictability, they are monotonous, flat and unvaried: changes are within a certain range, data tracks a linear progress (time data), develops very slowly (temperature or humidity data), or in a condition can only change in a very limited range (sound or activity data). We framed the personal narratives in a comparable way: every morning 8 o'clock, we have breakfast; we walk with our dog in the same park twice every day; we cook food every two days; we turn to video streams every night after work and we know we always can laugh when looking at Best Comedy Shows. In this iteration, multiple narratives were scripted in such a way that they could be combined as a representation of distinct types of everyday data.

2.1.2 Desired experience. Drafting the desired experience, we defined that dynamics of environmental data and changes of human movements both would allow visitors to discover in their experiences. Once visitors enter the space, they should immediately get the feeling that their presence was captured by the installation. Simultaneously, they should discover that they can influence some aspects of the installation experience by their proximity to it and by being in the space, whereas some other aspects are unaffected and independently evolve over time. The visitors would eventually also notice (1) the difference between the input data, (2) the connection between different data types and the content of the videos, and (3) the reaction of the installation to changing data values.

\subsection{Iteration 2: introducing data scenarios and two translations}

In the second iteration, we introduced the concept of data scenarios and used both data scenarios and personal narratives to strengthen the connection between everyday data and video vignettes: a personal narrative was combined multiple active data scenarios at a time and it was then visualized by a set of video vignettes [2] played in sequence (Figure 2).

2.2.1 Translation to data scenarios. In this iteration, we defined data scenarios as simplified indicators of changes in data. Temperature and humidity data were categorized by their relative changes (higher, same, or lower) and then included the same three scenarios as "increase", "non-change", and "decrease". Time data was categorized by different value ranges during 24 hours. Therefore, in our time data, four scenarios - "non-active", "active", "drinking", and "eating" -were connected as a continuous series of events in a 24-hour time schedule. Sound (loud or non-loud), proximity (close or non-close), and presence data (present or non-present) were categorized by each two different ranges of value and all included two scenarios as "active" and "non-active". Those data scenarios were specific to a data type, i.e., at any time, six data scenarios would be active and provide a rough contextual summary, the current personal narrative. For instance, at noon (time data approaching $12 \mathrm{pm}$, i.e., lunch time), the actor in the video would behave similar to visitors were they at home: eat, drink and relax.

2.2.2 Translation from data scenarios to video vignettes. The data scenarios allowed us to script and film the video vignettes more accurately and better aligned with the data scenarios and the personal 


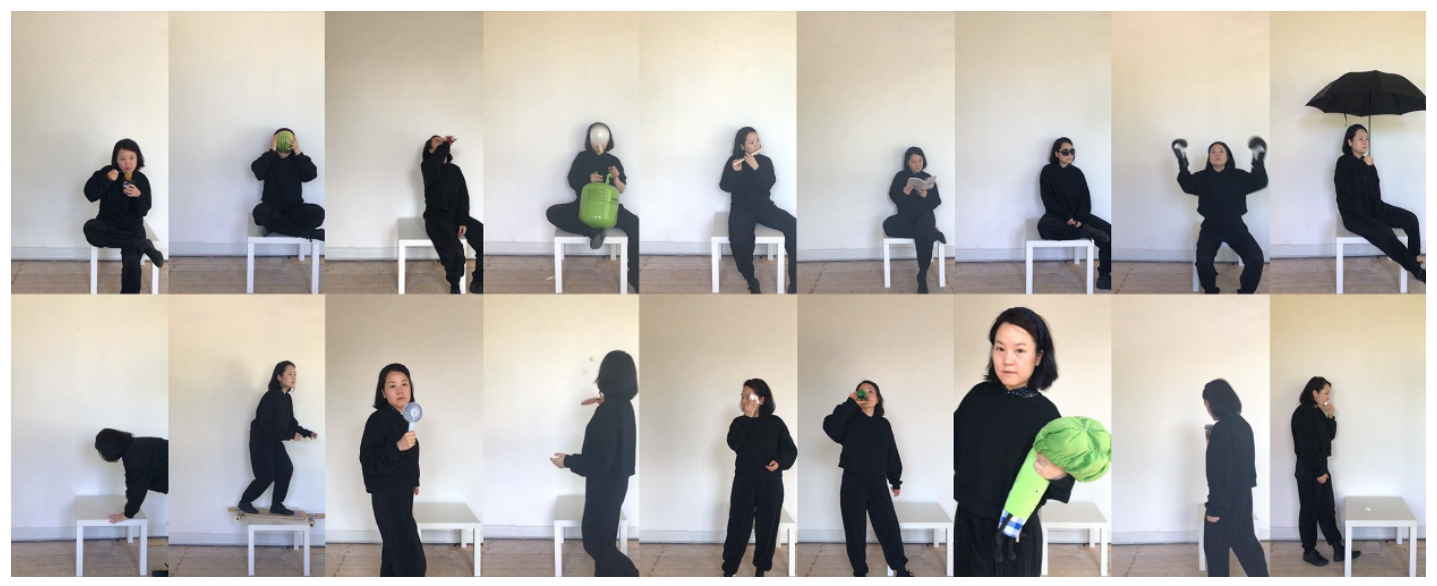

Figure 4: The use of different props helped visitors understand the narratives in a glimpse and connect narratives with the data information.

narratives they would prescribe. The video vignettes that expressed the personal narratives were designed by scripting them as data scenarios and then recording them according to the script. When scripting the video vignettes, we were inspired by the scenery settings, structural properties, and costume design of future smart home systems and their performativity as portrayed in the Black Mirror "White Christmas" episode (2014) ${ }^{1}$. We staged our narratives in a used environment and minimized the changes for the full set of video vignettes, and same for the avatar's costumes and facial expressions when performing in the videos.

We also polished the design of different body languages to build connectivity between data scenarios and video vignettes. We considered the use of three elements: movement, gesture, and eye contact in narratives to support different data scenarios. For instance, within the time data, we designed the single dull gesture with minimal movements on the stage for the "non-active" scenarios, and a flashed figure jumping across the stage for the "active" scenarios (Figure 3). Besides, we designed narratives with and without eye contact to make the design choices for videos relating to the data scenarios - the "active" scenarios in proximity, and presence data. For example, the avatar in the video vignettes would make more eye contact if visitors stayed longer and continued moving around in the space.

We deliberately designed the video vignettes as minimal, focused approximations of the data scenarios, mostly just acted, sometimes with the help of props that had typical characteristics and could help visitors understand of the personal narratives instantly (Figure 4). The props were used to complement the body language, so the video would feel more authentic and intuitively relatable - almost as if the narratives were taken from the lives of the visitors. For instance, walking around wearing sunglasses would hint at an "increase" in temperature data and an "active" time of the day. The video vignette of "inflating the balloon until the balloon explodes", as another example, represents "active" sound data scenario.

\footnotetext{
${ }^{1}$ Black Mirror "White Christmas" Episode: https://www.imdb.com/title/tt3973198/
}

\subsection{Iteration 3: polishing details of data processing}

In the third iteration, we focused on the details of data processing in the installation, essentially gathering raw data from different sources, i.e., the environment or external sources, followed by preprocessing and categorizing towards data scenarios. As a second point of focus, we designed a mechanism to prioritize scenarios in the personal narrative and then select and queue a select number of video vignettes for spatial playback (Figure 2).

2.3.1 Integrating raw data, processed data to data scenarios. We first classified raw data in the installation as sensor data (sound, proximity, and presence data), time data, and external data (temperature and humidity data). For sensor data, we first defined the expected domain of the data as a range. Then we divided this range into two or three segments which served as a mapping of the sensor data to a categorical scale. For example, raw sound data was mapped to the "active" sound data scenario when the level of volume was exceeding $70 \mathrm{db}$, and to "non-active" otherwise. Proximity data was mapped to "active" only when the distance value was less than $30 \mathrm{~cm}$. The presence data scenarios required more complex processing: we used a camera in the installation and background subtraction followed by blob detection, and finally counted the blobs. For the time data scenarios, we refined the time slots on a 24-hour basis (Figure 5a). Finally, for the external (weather) data, we defined different categories according to the changes in temperature or humidity data. After this step, we obtained processed data in categories which could be mapped to data scenarios per data stream. We designed an information interface (Figure 6a) to allow visitors to look behind the scenes of data processing. This interface was eventually shown in a kiosk app on an iPad.

2.3.2 Scheduling data scenarios in priority, selection and randomization. A second challenge in this iteration was that data scenarios would switch at different rates (weather and time data slowly; sound, proximity and presence data faster). The data scenarios were assigned a priority level based on their change rates (from fast to slow) - first sensor data, then time data and finally external data. 


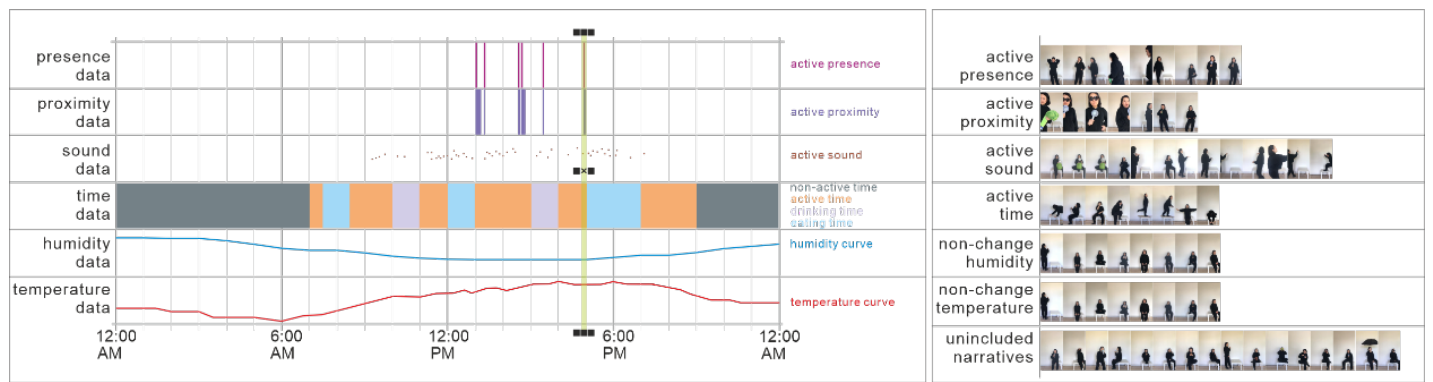

Figure 5: From left to right: (a) illustration of six types of data scenarios in a 24-hour time scale. The vertically highlighted part is the selected time slot which combines the scenarios at that time into a personal narrative, (b) the choice of video vignettes for each data scenarios at a time slot.

We gave sensor data a much higher priority than the other two data types because we intended to allow for an instant reaction to visitors' presence and their interaction, and engage them by updating data scenarios fast. Time data followed a predefined 24-hour time schedule. External data changed much slower and would have little influence on the personal narrative and selection of videos. We mapped the different data streams to different scenarios flowed that were sampled to obtain a six-dimensional snapshot (Figure 5a). This scenario snapshot was used to trigger the selection of video vignettes at any given moment (Figure $5 \mathrm{~b}$ ). Also, the video vignettes would need to be selected in a way that would give priority to faster changing scenarios than to others. For example, at noon time, sound or presence should be given priority instead of time, because these scenarios changed faster and thus resulted in more variation in the video vignettes. The choice of video vignettes was based on the different streams of data scenarios with priority given to presence, proximity, and sound.

2.3.3 Selection of video vignettes for a narrative. Given the abundance of filmed videos, a personal narrative, derived from the six overlapping active data scenarios at any given moment, was linked to more videos than what could be shown in the expected total runtime of the personal narrative (eventually the data would change and trigger a different personal narrative). The video vignettes were selected proportional to their priority: $50 \%$ from the scenarios with first priority, then $30 \%$ from the second, and so on, until up to a sequence of 20 video vignettes to be shown sequentially at a time. The selection process was otherwise randomized, i.e., the same personal narrative could result in different videos to be shown. We tested different refresh rates and decided on a timeout of 20 seconds for every update of the presence data. During this timeout, no data changes would be honored.

\subsection{Implementation}

After three design iterations, the installation was built for a ten-day public exhibition in a $24 \mathrm{~m}^{2}$ indoor space. In the installation setup, both backstage (iPad showing data mapping, large screen showing the full scheduling of videos as tiles) and frontstage were combined to allow visitors to experience and inspect the metamorphosis from raw data to video vignettes. We displayed an information interface updating six types of data, a tiled video overview with all video vignettes (queue with selected video selections, remaining videos), video vignettes. The implementation will be in two parts: three technology platforms and the distributed system.

2.4.1 Technology platforms. Data collection and processing required different technology platforms. In our case, there were three technology platforms to build up the interaction - (1) an iPad running a digital interface as the display for internal data (also providing time data) (Figure 6a), (2) a Raspberry Pi 4 single-board computer $(\mathrm{SBC})$ was running a central server and receiving updated data from external APIs (temperature and humidity data) and WIFI sensor shields (sound and proximity data), (3) a second SBC was connected to a WIFI router, processed a camera feed with a Python script (for presence data). This SBC was connected to a monitor to display all 65 video vignettes with the first 20 videos in an ordered queue (Figure 6b). This first video vignette in the queue represented the currently active personal narrative which was displayed on a monitor in portrait orientation (Figure 6c) connected with the first $\mathrm{SBC}$ and also on an iPod touch with the same portrait orientation. We suggested visitors to bring this mobile device with them if they were moving to other exhibition rooms.

2.4.2 Distributed system for data flow. The overall installation was implemented as a distributed system, using a design middleware [15] to connect all components of the system which were implemented on suitable platforms. The distributed system allowed us to move any aspects of data processing to an opportune component rather than being restricted to a singular central processing component. The system connected data from an external weather API, from two sensor data collectors based on ESP32 boards (sound and proximity data) and distributed the data to different visual components. The information interface (Figure 6a) received the raw data and visualized the internal data processing in a scrolling list of text for each block of data. Figure $6 \mathrm{~b}$ shows the Processing based overview of all 65 video vignettes with a priority order. This video overview would refresh its order and the queue of current video vignettes (i.e., matching the active personal narrative) with updated data. Figure $6 \mathrm{c}$ shows one of the video vignettes in portrait orientation. In the distributed system, the iPad interface shown in Figure $6 \mathrm{a}$ controlled the data processing and scenario mapping as well as the selection of video vignettes according to the current 


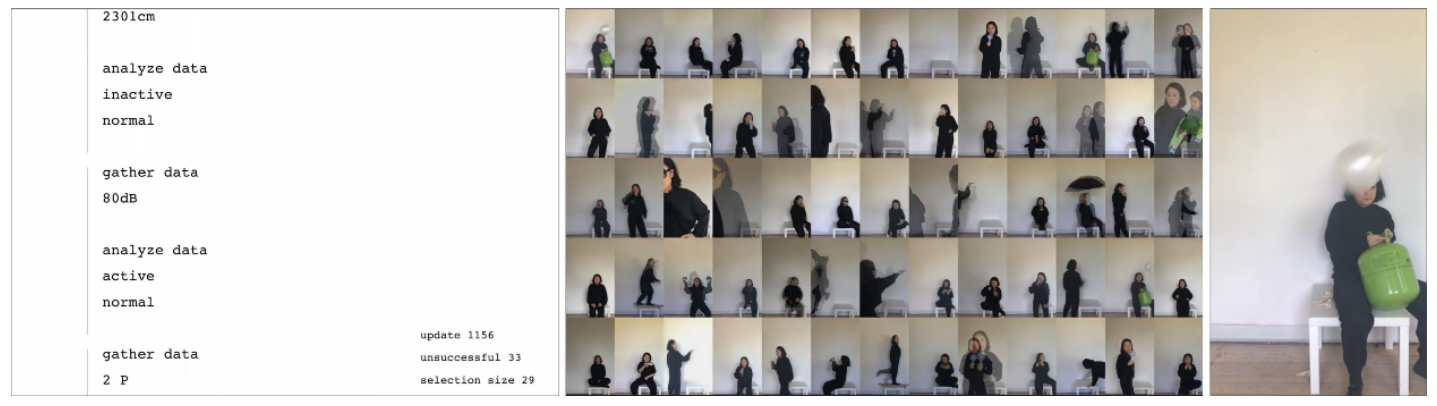

Figure 6: From left to right: (a) a digital interface showing internal data processing (iPad), (b) Processing-based tile view showing all 65 video vignettes (large screen), (c) individual video vignettes in portrait display (smaller screen, iPod touch).

personal narrative, and the SBC with the Processing tile view scheduled the video playback. Data sensing worked independently on three platforms.

\section{DISCUSSION}

We present feedback and insights from the exhibition led by a discussion of the installation and design process in five themes: (1) understandability and approachability, (2) relations between narratives and everyday data, (3) slow design, (4) subjectivity and interpersonal relationship, and (5) the iterative data design process. While the first four themes draw on our account of the visitor experiences and a post-hoc reflection on the installation design, the last theme stems views the entire process from inception to the setup in the exhibition space through the lens of data design.

\subsection{Understandability and approachability}

The installation did not provide hints about how visitors should approach it and the occasionally delayed responses left some visitors wondering about the connection between their presence or their actions and the responses from the installation. Technology-affine visitors were sensitive about the scattered sensors in the space and they tried to interact and to see how the output in the installation would change. Other visitors did not understand the interface of internal data information: "The iPad seems like displaying some numbers." These visitors paid more attention to the content of the video vignettes and their changes. We received similar comments from this group of visitors such as "The videos are funny." and "The order of videos was refreshed like following some kinds of rules." The content in the video vignettes indeed stood for the complexity of data scenarios, but this complexity also affected "drawing a clear boundary between a subjective account and a misrepresentation" [8]. On the other hand, the iPod touch, which was suggested to pick up and bring along when visiting other installations in the exhibition, helped the visitors focus on a single video vignette instead of becoming entangled in a complex network of data and interactions in their experiences. Besides, oral explanations in the exhibition also helped visitors connect with the installation at a deeper level than just with the videos. Some visitors returned to the installation or stayed for a longer time after receiving the explanations. Nevertheless, the exhibition setup showed that visitors needed a prior explanation to make sense of the different stages that were presented next to each other: the two single-narrative displays as the front stage, the large display with all narratives as a form of orchestration stage, and the single iPad display next to the sensors as a backstage that formed the connection to real-world phenomena and sensed signals. This exhibition design provided visitors with direct and unmediated access to every stage at once, and left them to their own intuition and interest in how this would approach the different artefacts of the project. Given a different exhibition space, an alternative setup using multiple rooms or a single room with dividers could isolate and focus the visitor's attention on particular stages, which could then be presented in a predetermined sequence from front stage to sensor stage to the orchestration. As the conversations with visitors showed, the conceptual depth of the installation is significant, and the alternative approach and setup would have probably led to a clearer visitor experience. Given the relatively short attention span of visitors touring a larger exhibition with multiple installations and artworks, additional guidance would help visitors make better sense of the installation facets and form a more cohesive picture, shifting questions from how and what to the why. A second reflection concerns the conceptually close connection between the front stage and the Everyday as represented by the exhibition room. This embedding and integration of the front stage into a lived environment as distributed displays of varying sizes and packaging corresponds to the initial thoughts and motivation of the installation. This was less visible in the final exhibition setup, where visitors could not easily make the switch from visiting an everyday place to the attempt to live in it for a brief period. The alternative exhibition setup as described above would have supported this, through a self-contained front stage with additional furnishing and decorating.

\subsection{Relations between everyday data and the narratives}

When starting to design this installation, we were curious about new ways to understand and mediate our relationship with everyday data. We combined (1) video as the medium and (2) personal narratives relating to the subject of everyday data. Muted video was used as an effective medium to address and deliver the abstract data in detail. At the same time, we were intrigued by the use of continuous video feeds in the periphery of the Everyday, embedded in personal living spaces, on tables, on walls and in unexpected corners. Repetitive videos, especially with intentionally rather mundane content, promised a balance between potential for engagement 
and visual ambiance. At the same time, repetitive videos are a reference (and nod) to animated GIFs that we see in abundance on social media nowadays-often cut-outs of movie scenes that isolate and further compress a mood, meme or interaction. Vignettes of a life online. The conceptual step to integrating newly produced video narratives into the physical Everyday was small. The personal narratives as a second aspect connected the vignettes to the data, but in a form that would not emphasize or even hint at abstract data. Instead, the installation connected the visitors to what the data was about, a situation or prediction in real-life. In other words, we took data entirely out of the equation, and designed the installation around visual prompts of what was happening. Although we also provided the backstage of the data processing in the iPad and Processing visualization, we observed that visitors preferred watching the videos over reading rolling-in numbers and texts. Videos were clearly adding to their experiences by being recognizable and relatable, but not "spelling out" real-life phenomena. Indeed, the videos were not meant to be an equivalent to printing " $32\left\{^{\circ}\right\} \mathrm{C}$, sunny, nobody else at home, time for lunch". Instead, the 65 video vignettes contained a diverse selection of content that was filmed to be neutral, yet composable and indirectly connected to the processed data. This would allow visitors to triangulate the meaning. It was more important for us to redesign the experience of everyday data than to optimize for comprehension, interpretation and action. Although this could not be fully confirmed in ten exhibition days, we were interested in the long-term appeal of the video vignettes as rich, peripheral information decorations [3].

\subsection{Slow design}

A clear challenge was that video as a medium can be distracting [11] because of, e.g., fast changing content, distracting elements or behavior, or misaligned content with respect to the processed data. Accordingly, we staged and filmed the video vignettes in a neutral setting with few props and almost no "breaking of the fourth wall". The brevity of clips and their random order turned out to be beneficial for this effect. A crucial point here is that slow technology or slow design does not necessarily mean to design with slow dynamics or smooth movements. Slow design is about the effect and experience that such a design evokes. Peripheral, repetitive video content in loops and with low engagement can have the same effect and should be(come) a part of the slow design toolbox (again, there is a considerable gap in research). The exhibition setup counteracted this design intention: by presenting the three stages of the installation together and in connection, the aesthetics of slow design were diluted or even overshadowed by the busyness of the backstage and orchestration display which changed rapidly and often even after seconds of stabilizing. This points at the tension between serendipity and responsiveness that we aimed to balance in the installation: on the one hand, we wanted to achieve an almost timeless quality of being immersed in the Everyday, and on the other hand, the installation had to react prompt to changes in the underlying data-in part reacting to visitors' movements, proximity and utterances.

\subsection{Subjectivity and interpersonal relationship}

The expression of subjectivity in the visuals led to a loss of the interpersonal relationship [8], which might have counteracted continuity and how the vignettes helped visitors build a mental model of the real-life processes behind the data. Some particularly "active" narratives could be recognized more easily by visitors, others remained ambiguous or vague. Although this balance between recognition and ambiguity contributes to letting the installation "grow" with a visitor, we do not understand well enough how to scale the effect and the data richness of the installation. This is a different notion than uncertainty in data visualization literature $[5,6,21,34]$, we explore to some extent the sense-making of narratives acted by another person while attempting to make an over bigger leap from felt experience to narrated mapping of sensed data to action. A future installation, potentially even without the backstaging of information processing might help clarify the connection between the content of narratives and the content of data in depth, and future research might play a role in further unpacking how the experience shown in the video vignettes can reflect underlying data.

\subsection{Data design in iterations}

The three iterations in the installation design and implementation show characteristics of a data-driven or data enabled process $[4,9,12,14]$, while also diverging in the focus on conceptual mapping: not the available data defines the outcomes, but the envisioned outcomes shapes the inclusion of data. We translated data to experiences by starting with conceptual definitions and placeholders, then iterating towards real-time data streams. In the first iteration we approached contextual data only conceptually and focused on personal narratives as a means to help visitors empathize with the real-life phenomenon behind the data (and not the data). After filming the first videos, we realized that without defined input data, further progress was difficult. This led to six types of input data, a few personal narratives and a large collection of filmed videos. The next challenge, towards the second iteration, was that the connection between everyday data and video vignettes was conceptually clear but far too vague to implement. Therefore, we introduced data scenarios as an intermediate concept to design a more in-depth mapping mechanism between data and the narratives. We also realized that "personal narratives" needed to be composites of multiple scenarios that each depended on a specific type of data. This led to the slightly more "synthetic", but also operational redefinition of the "personal narrative". While this allowed us to continue with the video and overall installation design, we found several intricate problems when building the data collectors and attempting to connect external APIs to the data scenarios. This was a point that could not be resolved through more engineering and programming, we needed to take a step back and work on organizing the data scenarios together with the videos: enter a large Excel sheet that not only pinpointed the most interesting ranges and value changes of raw data, but also their mapping toe scenarios and finally exhaustive lists of matching videos. Having this reference document proved to be crucial in the design and implementation of the control interface (iPad) and the Processing interface (big screen). 
Overall, the process was not as clearly split into design and implementation as Figure 2 might suggest. At the top level, our design process was dominated by balancing between accurate data presentation and visitors' overall experience, essentially a balance between understanding and empathy. Underneath, we iterated quickly between implementing smaller parts, then going back to design connections, then back to implement them or another component which in turn led to further needs to design. The installation was tested in the studio and built in the exhibition within hours without a need to adjustment or further tweaking. In hindsight, what surprised us was the importance of operational concepts throughout the design iterations, which triggered (heated) discussions and allowed us to explore the different aspects of data metamorphosis that were present in the final installation long before the installation was operational. As a last point of reflection, writing this case study paper was not planned from the begin, instead it emerged from our discussions and the realization that the conceptual richness in this project was worth documenting.

\section{CONCLUSION}

This case study reports on the design of an interactive installation that reflects on how mundane video vignettes can be used to mediate everyday data encounters. The work suggests that the visualization of everyday data can venture beyond numbers and charts as practiced as the current state of the art. Seeing the world through numbers affects the human viewer, it flattens their perception. There are risks involved when we introduce data representations in the Everyday: in our urge to inform and inform even more we superimpose a flattened perspective on all the things, events and their interconnections around us. When setting sights on the Everyday in this project, we let go of effectiveness and practicality, and explored how to project mundane data into a daily context using narrative forms - essentially taking the data out of the spotlight and into a backstage. Our results show a new form of visualizing and contextualizing everyday data as a data-driven, but not data-mediated mapping between real-life phenomena (encoded by different types of raw data) and visual narratives that let a viewer triangulate back to the phenomena in context. The installation was exhibited for ten days and indicates that visitors could relate well to the video vignettes. Although it is difficult to further generalize based on a ten-day public exhibition, this exhibition allows us to speculate about future smart home data visualization approaches that follow a similar translation approach.

A second contribution in this case study is the layered process that features concepts that are expanded from iteration to iteration. While this process is by far not a blueprint, or even a method, it shows how an artistic project that starts with a strong core vision can incrementally proceed towards implementation with frequent switches between design and engineering. This project had several turning points (see discussion of the design process), where we usually fell back to the core vision and guiding principles. We hope that this report of an open process experience can benefit others in the community who face open-ended, data-entangled, conceptually rich design projects. In the future, we aim to extend our project into a long-term study in several everyday contexts to investigate other emerging applications, e.g., integrating a single video vignette as a mural or as a smartphone application reacting on localized events, and to investigate its qualities and a broader range of aesthetics in informing and redirecting attention in the Everyday.

\section{ACKNOWLEDGMENTS}

The installation "Lost in Illusion" was the result of a summer artist residency program at the Albert van Abbehuis in 2020, and it was exhibited from August 27 till September 6, 2020 in the context of the group exhibition "POLATECH 1". We thank Tom Veeger, MAD emergent art center and Cultuur Fonds Eindhoven for their kind help and support.

\section{REFERENCES}

[1] Michael Alles and Miklos A. Vasarhelyi. 2014. Thick data: adding context to big data to enhance auditability. International fournal of Auditing Technology 2, 2: 95-108.

[2] Irmgard Bartenieff and Dori Lewis. 1980. Body movement: Coping with the environment. Psychology Press.

[3] Genevieve Bell, Mark Blythe, and Phoebe Sengers. 2005. Making by making strange: Defamiliarization and the design of domestic technologies. ACM Transactions on Computer-Human Interaction (TOCHI)12, 2: 149-173.

[4] Sander Bogers, Janne van Kollenburg, Eva Deckers, Joep Frens, and Caroline Hummels. 2018. A situated exploration of designing for personal health ecosystems through data-enabled design. In Proceedings of the 2018 Designing Interactive Systems Conference, 109-120.

[5] Georges-Pierre Bonneau, Hans-Christian Hege, Chris R Johnson, Manuel M Oliveira, Kristin Potter, Penny Rheingans, and Thomas Schultz. 2014. Overview and state-of-the-art of uncertainty visualization. In Scientific Visualization. Springer, 3-27.

[6] Ross Brown. 2004. Animated visual vibrations as an uncertainty visualisation technique. In Proceedings of the 2nd international conference on Computer graphics and interactive techniques in Australasia and South East Asia, 84-89.

[7] Mackinlay Card. 1999. Readings in information visualization: using vision to think. Morgan Kaufmann.

[8] Sheelagh Carpendale, Alice Thudt, Charles Perin, and Wesley Willett. 2017. Subjectivity in personal storytelling with visualization. Information Design fournal 23, 1: 48-64.

[9] Elizabeth F Churchill. 2016. Designing Data Practices. interactions 23, 5: 20-21. https://doi.org/10.1145/2983401

[10] Rogerio De Paula, Xianghua Ding, Paul Dourish, Kari Nies, Ben Pillet, David F Redmiles, Jie Ren, Jennifer A Rode, and Roberto Silva Filho. 2005. In the eye of the beholder: a visualization-based approach to information system security. International fournal of Human-Computer Studies 63, 1-2: 5-24.

[11] Semih Delil. 2017. The impact of infographic animation videos on data visualization. International Journal of Social Sciences and Education Research 3, 4: 1178-1183.

[12] Melanie Feinberg. 2017. A Design Perspective on Data. In Proceedings of the 2017 CHI Conference on Human Factors in Computing Systems (CHI '17), 2952-2963. https://doi.org/10.1145/3025453.3025837

[13] Pepijn Fens and Mathias Funk. 2014. Personal Health Data: Visualization Modalities and Their Perceived Values. In Proceedings of the 22nd International Conference in Central Europe on Computer Graphics, Visualization and Computer Vision 2014, 339-344.

[14] Mathias Funk. 2016. Design and data: Strategies for designing information products in team settings. In Collaboration in creative design, Springer, Cham, 351-378. https://doi.org/10.1007/978-3-319-29155-0_17

[15] Mathias Funk. 2019. OOCSI. https://doi.org/10.5281/zenodo.1321219

[16] Mathias Funk, Lin-Lin Chen, Shao-Wen Yang, and Yen-Kuang Chen. 2018. Addressing the Need to Capture Scenarios, Intentions and Preferences: Interactive Intentional Programming in the Smart Home. International fournal of Design 12, 1.

[17] Henning Griethe, Heidrun Schumann, and others. 2006. The visualization of uncertain data: Methods and problems. In SimVis, 143-156.

[18] Barbara Grosse-Hering, Jon Mason, Dzmitry Aliakseyeu, Conny Bakker, and Pieter Desmet. 2013. Slow design for meaningful interactions. In Proceedings of the SIGCHI Conference on Human Factors in Computing Systems (CHI '13), 3431-3440. https://doi.org/10.1145/2470654.2466472

[19] Lars Hallnäs and Johan Redström. 2001. Slow technology - Designing for reflection. Personal and Ubiquitous Computing 5, 3: 201-212. https://doi.org/10.1007/ PL00000019

[20] Yvonne Jansen, Pierre Dragicevic, Petra Isenberg, Jason Alexander, Abhijit Karnik, Johan Kildal, Sriram Subramanian, and Kasper Hornbæk. 2015. Opportunities 
and challenges for data physicalization. In Proceedings of the 33rd Annual ACM Conference on Human Factors in Computing Systems, 3227-3236.

[21] Amit Jena, Ulrich Engelke, Tim Dwyer, Venkatesh Raiamanickam, and Cecile Paris. 2020. Uncertainty Visualisation: An Interactive Visual Survey. In2020 IEEE Pacific Visualization Symposium (PacificVis), 201-205.

[22] Rohit Ashok Khot, Jeewon Lee, Larissa Hjorth, and Florian'Floyd' Mueller. 2014 SweatAtoms: understanding physical activity through material artifacts. In CHI'14 Extended Abstracts on Human Factors in Computing Systems. 173-174.

[23] Lenneke Kuijer and Annelise De Jong. 2012. Identifying design opportunities for reduced household resource consumption: exploring practices of thermal comfort. Fournal of Design Research 1410, 1-2: 67-85.

[24] Kristin M Langellier. 1989. Personal narratives: Perspectives on theory and research. Text and Performance Quarterly 9, 4: 243-276.

[25] Charlotte Linde and others. 1993. Life stories: The creation of coherence. Oxford University Press on Demand.

[26] Deborah Lupton. 2018. How do data come to matter? Living and becoming with personal data. Big Data \& Society 5, 2: 2053951718786314. https://doi.org/10.1177/ 2053951718786314

[27] Ezio Manzini and John Cullars. 1992. Prometheus of the Everyday: The Ecology of the Artificial and the Designer's Responsibility. Design Issues 9, 1: 5-20.

[28] Jimmy Moore, Pascal Goffin, Miriah Meyer, Philip Lundrigan, Neal Patwari, Katherine Sward, and Jason Wiese. 2018. Managing in-home environments through sensing, annotating, and visualizing air quality data. Proceedings of the ACM on Interactive, Mobile, Wearable and Ubiquitous Technologies 2, 3: 1-28.

[29] Stanislaw Nowak, Lyn Bartram, and Thecla Schiphorst. 2018. A MicroPhenomenological Lens for Evaluating Narrative Visualization. In2018 IEEE Evaluation and Beyond-Methodological Approaches for Visualization (BELIV), 11-18.

[30] William Odom, Richard Banks, Abigail Durrant, David Kirk, and James Pierce. 2012. Slow technology: critical reflection and future directions. In Proceedings of the Designing Interactive Systems Conference (DIS '12), 816-817. https://doi.org/ $10.1145 / 2317956.2318088$

[31] Caroline Overgoor and Mathias Funk. 2018. IdleBot: Exploring the Design of Serendipitous Artifacts. In DIS 2018 Companion - Proceedings of the 2018 ACM Conference on Designing Interactive Systems.

[32] Bruno Pagno and Luciana Nedel. 2015. Everyday Visualization. In Electronic proceedings of the IEEE VIS 2015 workshop Personal Visualization: Exploring Data in Everyday Life, 1-4.

[33] Alexandros Pantelopoulos and Nikolaos G Bourbakis. 2009. A survey on wearable sensor-based systems for health monitoring and prognosis. IEEE Transactions on Systems, Man, and Cybernetics, Part C (Applications and Reviews)40, 1: 1-12.
[34] Binh Pham, Alex Streit, and Ross Brown. 2009. Visualisation of Information Uncertainty: Progress and Challenges. In Trends in interactive visualization. Springer, 19-48.

[35] James Pierce. 2019. Smart home security cameras and shifting lines of creepiness: A design-led inquiry. In Proceedings of the 2019 CHI Conference on Human Factors in Computing Systems, 1-14.

[36] Sarah Pink, Debora Lanzeni, and Heather Horst. 2018. Data anxieties: Finding trust in everyday digital mess. Big Data \& Society 5, 1: 2053951718756685. https: //doi.org/10.1177/2053951718756685

[37] Zachary Pousman, John Stasko, and Michael Mateas. 2007. Casual information visualization: Depictions of data in everyday life. IEEE transactions on visualization and computer graphics 13, 6: 1145-1152.

[38] Amon Rapp and Federica Cena. 2016. Personal informatics for everyday life: How users without prior self-tracking experience engage with personal data. International fournal of Human-Computer Studies 94: 1-17.

[39] Tom A Rodden, Joel E Fischer, Nadia Pantidi, Khaled Bachour, and Stuart Moran. 2013. At Home with Agents: Exploring Attitudes Towards Future Smart Energy Infrastructures. In Proceedings of the Twenty-Third International foint Conference on Artificial Intelligence (IJCAI '13), 3057-3061. Retrieved from http://dl.acm.org/ citation.cfm?id=2540128.2540577

[40] Neelima Sailaja, Andy Crabtree, James Colley, Adrian Gradinar, Paul Coulton, Ian Forrester, Lianne Kerlin, and Phil Stenton. 2019. The living room of the future. In Proceedings of the 2019 ACM International Conference on Interactive Experiences for TV and Online Video, 95-107.

[41] Arvind Satyanarayan and Jeffrey Heer. 2014. Authoring narrative visualizations with ellipsis. In Computer Graphics Forum, 361-370.

[42] Kim Sauvé, Steven Houben, Nicolai Marquardt, Saskia Bakker, Bart Hengeveld, Sarah Gallacher, and Yvonne Rogers. 2017. LOOP: A physical artifact to facilitate seamless interaction with personal data in everyday life. In Proceedings of the 2017 ACM Conference Companion Publication on Designing Interactive Systems, 285-288.

[43] Ben Shneiderman, Catherine Plaisant, and Bradford W Hesse. 2013. Improving healthcare with interactive visualization. Computer 46, 5: 58-66.

[44] Tobias Skog, Sara Ljungblad, and Lars Erik Holmquist. 2003. Between aesthetics and utility: designing ambient information visualizations. In IEEE Symposium on Information Visualization 2003 (IEEE Cat. No. 03TH8714), 233-240.

[45] Fernanda B Viégas and Martin Wattenberg. 2007. Artistic data visualization: Beyond visual analytics. In International Conference on Online Communities and Social Computing, 182-191.

[46] Ron Wakkary, Doenja Oogjes, Henry WJ Lin, and Sabrina Hauser. 2018. Philosophers living with the tilting bowl. In Proceedings of the 2018 CHI Conference on Human Factors in Computing Systems, 1-12. 